\title{
P7-22 Troubles trophiques
}

\author{
C Springer-Verlag France 2013
}

\author{
P7-22-01 \\ Problématique de la fiche pansement dans le suivi \\ des plaies chroniques en hospitalisation \\ I. Mensah*, C. Menant, S. Roussel, \\ Groupe de travail des infirmières du SSR \\ Service de soins de suite réadaptation, hôpital de Saint-Marcellin, \\ Saint-Marcellin, France
}

Introduction : Dans le service de soins suite et rééducation, 20 à $30 \%$ des patients présentent des plaies chroniques. Leur cicatrisation optimale nécessite une réévaluation régulière et une transmission objective des données avec une traçabilité sur la fiche pansement-tâche chronophage. Afin que les infirmières se l'approprient dans leur fonctionnement hospitalier multitâche, cette fiche doit être adaptée, simple et objective. En effet, les items retenus sur ces fiches ne sont pas toujours exploités car incomplets ou absents, entravant ainsi le suivi et la prise en charge de ces plaies chroniques. Pour les infirmières, la fiche pansement doit être simple, complète, précise, objective mais facile et rapide à remplir !

Objectifs : Analyser les difficultés en lien avec l'application des items retenus sur une fiche pansement en hospitalisation afin de la rendre objective dans le choix du pansement et simple d'utilisation dans le suivi des plaies chroniques.

Méthodes : Une fiche pansement des plaies chroniques élaborée avec l'infirmière référente pansement a été évaluée après trois mois d'utilisation par toutes les infirmières du service. Celles-ci ont été au préalable formées aux différents items retenus. L'évaluation de la fiche s'est faite par un questionnaire écrit anonyme, un entretien individuel puis collectif afin d'avoir le ressenti de chacune.

Résultats : Toutes les infirmières du service trouvent la fiche pansement facile à remplir et simple ; les items retenus sont connus de toutes. Néanmoins, $30 \%$ d'entre elles expriment le besoin d'un rappel oral régulier par l'infirmière référente sur les notions d'aspect de l'état de la plaie et celui de la peau périphérique. En effet, ces items restent complexes et absents dans $30 \%$ des fiches. La formation initiale et continue devrait permettre de rendre ces items plus accessibles et donc mieux tracés et exploitables. Les codes couleur ou les abréviations ne sont utilisés que par $50 \%$ des infirmières. Pour toutes, demeure la problématique des dimensions des lésions multiples et/ou irrégulières, des outils de mesure pour les lésions excédant $15 \mathrm{~cm}$, et de la fréquence des mesures. L'utilisation de calque permet un suivi visuel global mais nécessite du temps pour être réalisé. Pour toutes, les transmissions écrites restent importantes et nécessaires bien que limitées et précises car le besoin de s'exprimer demeure. Cet item leur permet également de noter les éléments non identifiés telles la notion de douleur, la réalisation d'un prélèvement. Le choix du pansement est mieux adapté, plus conforme, et le suivi plus objectif avec une transmission plus expressive. Dix pour cent des soignants ne remplissent pas la fiche après chaque pansement, entraînant ainsi une perte ou une imprécision des informations.

Conclusion : La fiche pansement des plaies chroniques en hospitalisation doit rester simple, pratique, mais suffisamment explicite et objective pour guider le choix des pansements et assurer le suivi des plaies. L'efficacité de son application par les infirmières repose sur une bonne connaissance des items retenus par les formations initiale et continue. Le référent infirmier reste un pilier dans la pédagogie. L'implication des infirmières dans la réalisation de la fiche permet de l'adapter aux réalités du service afin de la rendre effective dans son utilisation, en assurer la transmission et optimiser la cicatrisation dans le temps.

Conflit d'intérêt : les auteurs déclarent ne pas avoir de conflit d'intérêt. 